\title{
Symptomatic Metastasis to the Pituitary Gland: A Report of Three Cases and Review of the Literature
}

\author{
Peter YM Woo ${ }^{1 *}$, Ronald Li ${ }^{1}$, Yung Chan ${ }^{1}$, Timothy SK Chan², Peter KH Pang ${ }^{1}$, Kwong-Yau Chan ${ }^{1}$ and John CK Kwok \\ ${ }^{1}$ Department of Neurosurgery, Kwong Wah Hospital, Hong Kong, China \\ ${ }^{2}$ Department of Pathology, Kwong Wah Hospital, Hong Kong, China
}

\begin{abstract}
Objective and importance: Symptomatic metastases to the pituitary gland are rare and often herald advanced systemic malignant disease. Distinguishing them from benign pituitary adenomas is challenging. The clinicoradiological features of three patients with pituitary metastases are presented. We also review the literature from 1970 to 2015 to formulate a diagnostic strategy.

Clinical presentation: All patients were elderly with ages ranging from 65 to 82 years old. One had a previously undiagnosed prostatic carcinoma and two had known histories of primary gastric or parotid salivary gland carcinoma. Rapid visual deterioration was the most common symptom followed by ophthalmoplegia. Cranial diabetes insipidus (CDI) was observed after administrating hydrocortisone replacement in one patient. All patients had evidence of a solitary pituitary tumor on magnetic resonance imaging (MRI) of which two had discernible posterior pituitary signal changes. Trans-sphenoidal excision was performed for all patients with no procedure related complications. Two patients had visual improvement, but due to advanced systemic disease all succumbed within two months. A literature review of 475 pituitary metastases revealed that breast $(37.1 \%)$ and bronchogenic carcinomas $(22.1 \%)$ were the most frequently encountered primary malignancies. Among 290 symptomatic patients $(22.1 \%)$ were the most frequently encountered primary malignancies. Among 290 symptomatic patients the commonest presentation was CDI (19.9\%) and 12.1\% had ophthalmoplegia. Of the 166 tumors with documented neuroimaging features, $31.3 \%$ had evidence of pituitary stalk thickening or enhancement followed by cavernous sinus invasion (30.1\%) or was dumbbell shaped (27.7\%).

Conclusion: Diagnosing metastatic pituitary tumors is important for the treating clinician in order to avoid unnecessary surgical excision in patients with limited life expectancy. Patients older than 60 years, or with a history of cancer, presenting with CDI or ophthalmoplegia are red flag clinical features. For these patients we recommend investigations for occult primary malignancy when early posterior pituitary involvement orcavernous sinus invasion is demonstrated on MRI.
\end{abstract}

Keywords: Pituitary gland; Metastasis; Carcinoma; Cranial diabetes insipidus; Magnetic resonance imaging

\section{Introduction}

Symptomatic metastases to the pituitary gland are rare and diagnosed in less than $1 \%$ of pituitary tumor surgical resections [1]. The last 20 years has witnessed an increasing incidence in the detection and treatment of pituitary metastatic tumors due to improved imaging techniques and cancer patient survival [1]. Currently the majority of metastatic tumors to the pituitary are diagnosed incidentally with fewer than $10 \%$ of patients being symptomatic [2]. Malignancies of essentially all tissue types have been reported, but the most commonly encountered is breast followed by bronchogenic carcinoma $[3,4]$. The clinic radiological features of three patients with symptomatic pituitary metastasis from carcinomas of the prostate, stomach and parotid gland are described. The last extensive review of the literature on pituitary metastases was performed more than a decade ago and we provide an updated summary of tumor origin, symptom presentation and Magnetic Resonance Imaging (MRI) features of reported cases from 1970 to 2015 $[3,4]$. Differentiating metastatic pituitary tumors from benign lesions is an important diagnostic challenge for the treating clinician in order to avoid undue surgical excision in patients with advanced stage disease. We propose a diagnostic strategy to raise clinical suspicion for these rare lesions.

\section{Case Reports}

\section{Patient 1}

A 65 year-old Chinese male presented with diplopia and left eye blurring of vision for one month. The Patient had left sixth nerve cranial palsy with a visual acuity of 20/70 and bitemporal hemianopia. MRI depicted a $3.8 \mathrm{~cm}$ heterogeneously contrast enhancing pituitary tumor with stalk thickening $(3.1 \mathrm{~mm})$, bilateral cavernous sinus invasion, encasement of the right carotid siphon and optic chiasm compression (Figure 1a and $1 \mathrm{~b}$ ). There was no other brain lesion detected. Endocrinological work-up revealed panhypopituitarism with mild hyperprolactinemia $(37.2 \mathrm{ng} / \mathrm{ml})$ (Table 1$)$. Trans-sphenoidal subtotal excision of the pituitary tumor was performed for optic nerve decompression. Intraoperatively a firm and vascular pituitary lesion was encountered. Postoperatively the patient had improved left visual acuity to 20/40, but he continued to have diplopia and bitemporal hemianopia. The final histopathology was metastatic adenocarcinoma of the prostate (Figure 1c and 1d). Subsequent serum prostatic specific antigen levels were elevated $(256 \mathrm{ng} / \mathrm{ml})$ and a whole-body positron emission tomography scan showed extensive aorto-caval lymph nodes and multiple vertebral bone metastases. Palliative craniospinal radiotherapy and Orchidectomy was performed, but the patient succumbed two months later.

*Corresponding author: Peter YM Woo, Hong Kong Room 318 Nursing Quarters Kwong Wah Hospital, 25 Waterloo Road, Hong Kong, Tel: 3517 5052, Fax: 3517 5260; E-mail: wym307@ha.org.hk

Received May 09, 2015; Accepted June 08, 2015; Published June 14, 2015

Citation: Peter YMW, Ronald L, Yung C, Timothy SKC, Peter KHP, et al. (2015) Symptomatic Metastasis to the Pituitary Gland: A Report of Three Cases and Review of the Literature. J Neurol Disord 3: 236. doi: 10.4172/2329-6895.1000236

Copyright: (c) 2015 Peter YMW, et al. This is an open-access article distributed under the terms of the Creative Commons Attribution License, which permits unrestricted use, distribution, and reproduction in any medium, provided the original author and source are credited. 

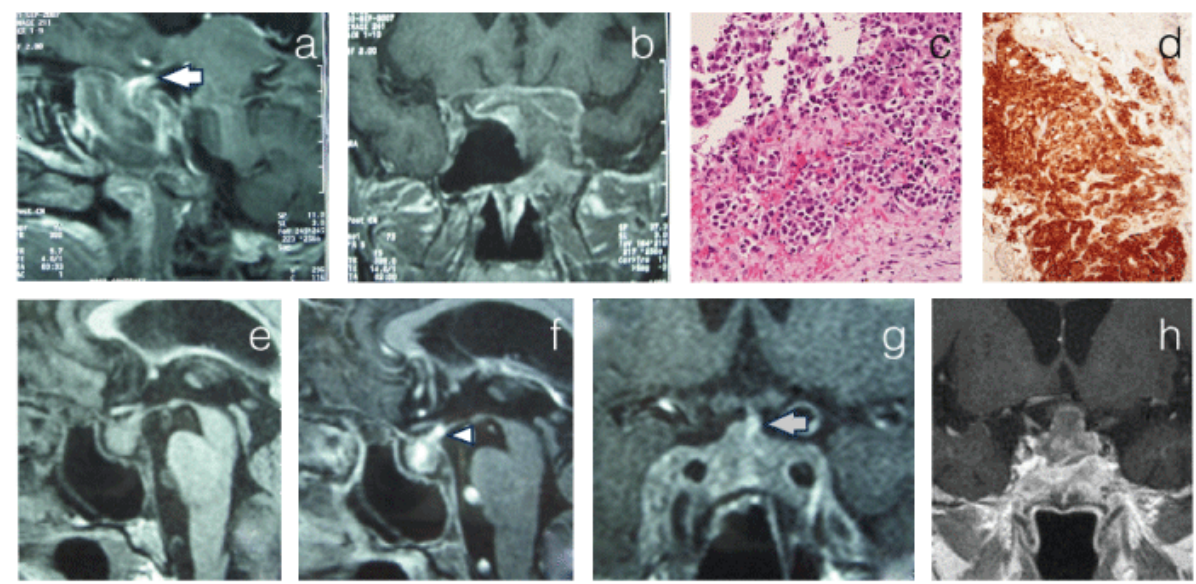

Figure 1: MRI of Patient 1 revealing a large pituitary tumor with invasion into the cavernous sinuses and contrast enhancement of the pituitary stalk (a) white arrow, T1W sagittal view; (b) T1W coronal view). Hematoxylin and eosin stain (c) of patient 1's pituitary lesion showing pleomorphic polygonal tumor cells with prominent nucleoli and frequent mitoses. Tumor cells were positive prostatic specific antigen, (d) MRI of Patient 2 depicting loss of the usual posterior pituitary hyperintense signal $€$ sagittal, T1W non-contrast enhanced) and avid stalk enhancement (f) white arrowhead, sagittal, T1W contrast enhanced; (g) grey arrow, coronal, T1W contrast enhanced). Pituitary tumor MRI of Patient 3 forming a dumbbell shaped mass commonly seen in metastasis (h) coronal T1W contrast enhanced).

\section{Patient 2}

An 82 year-old Chinese male, with a history of distal radical gastrectomy for stage IIa adenocarcinoma (T2, N1, M0 with clear margins) performed a year ago, experienced sudden headache for two days. Upon admission the patient was confused with orthostatic hypotension, but with intact visual acuity. MRI showed a single 1.2 $\mathrm{cm}$ isointense pituitary lesion with loss of the usual posterior lobe hyperintensity on T1-weighted sequence (Figure 1e). Contrast imaging revealed a heterogeneously enhancing pituitary tumor suggestive of intralesional hemorrhage with a thickened stalk of $3 \mathrm{~mm}$ (Figure if and g). Endocrinological work-up showed panhypopituitarism (Table 1) with extremely low morning serum cortisol levels ( $38 \mathrm{nmol} / \mathrm{l})$. After initiating intravenous hydrocortisone the patient developed polyuria, urine output was $1200 \mathrm{ml}$ over four hours, with low urine osmolality $(260 \mathrm{mOsm} / \mathrm{kg})$. There was associated hypernatremia $(155 \mathrm{mmol} / \mathrm{l})$ and Cranial Diabetes Insipidus (CDI) was diagnosed. Transsphenoidal tumor excision was performed and intraoperatively, the lesion had a fibrous pseudo-capsule with a soft core of avascular necrotic tissue. The final diagnosis was metastatic adenocarcinoma. The patient refused investigations for possible gastric carcinoma recurrence and adjuvant oncologic therapy. He succumbed three weeks after surgery

\section{Patient 3}

An 81 year-old Chinese female, with a history of left parotid salivary gland acinic cell carcinoma excised ten years ago, experienced progressive right eye blurring of vision for one month. Physical examination revealed right third nerve palsy, right blindness with optic atrophy. MRI showed a single $2.3 \mathrm{~cm}$ heterogeneously enhancing solid-cystic dumbbell shaped pituitary tumor with suprasellar extension. There was also infiltration to the right cavernous sinus and compression of the optic chiasm (Figure 1h). Hypothyroidism and mild hyperprolactinemia $(62.4 \mathrm{ng} / \mathrm{ml})$ was also detected. Transsphenoidal excision of the tumor was performed and intraoperatively a firm tan colored pituitary tumor compressing against the optic chiasm was observed. The pathological diagnosis was metastatic acinic cell carcinoma. Postoperatively the patient's vision failed to recover. Supportive care was offered in view of her advanced age and she passed away a month after surgery.

\section{Discussion}

The first reported case of metastasis to the pituitary gland was by Benjamin in 1857 discovered during an autopsy of a patient with disseminated melanoma [5]. The number of cases has increased ever since with presently more than 450 reported. Pituitary metastasis is uncommon and autopsy studies have quoted an incidence of between $1 \%$ to $3.6 \%$ in patients with known malignancy [4]. A certain degree of underreporting exists since most are asymptomatic and an estimated two-thirds of patients with latent pituitary metastasis have macroscopically normal-looking pituitary tissue that could easily evade detection during standard post-mortem examinations [2]. Metastasis typically occurs in older patients in the sixth to seventh decades of life with no apparent sexual predominance [3]. Our review of 475 reported cases from 1970 to 2015 shows that the commonest primary malignancies originate from either the breast $(37.9 \%)$ or lung $(22.8 \%)$ accounting for $60 \%$ of patients (Table 1). It is speculated that the predilection of breast cancer cells to metastasize to the pituitary may be due to the chemotactic and proliferative properties of prolactin $[3,6]$. Apart from malignant tissue origin, tumor location within the pituitary gland follows a distinct pattern. The majority of metastases involve the posterior lobe of the pituitary with only $13 \%$ to $15 \%$ of lesions occupying the anterior lobe $[3,4]$. Two reasons for this phenomenon have been suggested. First, the neurohypophysis is supplied directly by perforators of the internal carotid artery, namely the hypophyseal, infundibular and prechiasmal arteries. In comparison the adenohypophysis lacks an arterial supply and perfusion is indirectly derived from a portal venous system descending from the hypothalamus. It has therefore been postulated that hematogeneously spread tumor cells primarily deposit in the posterior pituitary and subsequently encroach the anterior lobe as the disease progresses $[3,4]$. Secondly, the posterior pituitary lobe has a wider area of contact with the sellar turcica and malignancies with a proclivity for bone metastases could spread contiguously via this route [4]. This could possibly explain why a disproportionate number of such tumors, namely prostatic, renal cell and thyroid carcinomas, together account for more than $10 \%$ of cases (Table 1 ).

Only $2.5 \%$ to $18.2 \%$ of pituitary metastases are symptomatic $[3,6]$. From our review of 290 patients, onefifth presented with CDI, followed by anterior pituitary insufficiency (16.3\%), visual field defects (15.9\%) and ophthalmoplegia (12.1\%) (Table 2). The high incidence of CDI reflects 
Citation: Peter YMW, Ronald L, Yung C, Timothy SKC, Peter KHP, et al. (2015) Symptomatic Metastasis to the Pituitary Gland: A Report of Three Cases and Review of the Literature. J Neurol Disord 3: 236. doi: 10.4172/2329-6895.1000236

Page 3 of 4

\begin{tabular}{|c|c|c|c|c|c|c|c|c|}
\hline Tumor origin & $\mathbf{n}$ & $\mathbf{\%}$ & Tumor origin & $\mathbf{n}$ & Tumor origin & $\mathbf{n}$ & $\%$ \\
\hline Breast & 176 & 37.1 & Leukemia & 5 & 1.1 & Germ cell tumor & 2 & 0.4 \\
\hline Lung & 105 & 22.1 & Liver & 5 & 1.1 & Ovary & 2 & 0.4 \\
\hline Gastrointestinal & 31 & 6.5 & Multiple myeloma & 5 & 1.1 & Thymus & 2 & 0.4 \\
\hline Prostate & 23 & 4.8 & Pharynx & 5 & 0.9 & Bile duct & 1 & 0.2 \\
\hline Kidney & 23 & 4.8 & Urinary bladder & 4 & 0.9 & Lymphosarcoma & 1 & 0.2 \\
\hline Unknown & 15 & 3.2 & Uterine cervix & 4 & 0.9 & Nasal cavity & 1 & 0.2 \\
\hline Thyroid & 14 & 2.9 & Larynx & 3 & 0.7 & Nasopharyngeal & 1 & 0.2 \\
\hline Melanoma/skin & 13 & 2.7 & Oral cavity & 3 & 0.7 & Penis & 1 & 0.2 \\
\hline Lymphoma & 8 & 1.8 & Paranasal sinus & 3 & 0.7 & Retroperitoneum & 1 & 0.2 \\
\hline Pancreas & 6 & 1.3 & Salivary gland & 3 & 0.7 & Rhabdomyosarcoma & 1 & 0.2 \\
\hline Endometrium & 5 & 1.1 & $\begin{array}{c}\text { Skin squamous } \\
\text { cell carcinoma }\end{array}$ & 3 & 0.7 & & & \\
\end{tabular}

Table 1: Primary malignancy origin of 475 reported pituitary metastases.

\begin{tabular}{|c|c|c|c|c|c|}
\hline Symptom/Finding & N & \% & Symptom/Finding & n & 5 \\
\hline Cranial diabetes insipidus & 120 & 19.9 & Decreased libido & 0.8 \\
\hline Anterior pituitary insufficiency & 98 & 16.3 & Seizures & 0.7 \\
\hline Visual field defect & 96 & 15.9 & SIADH & 3 \\
\hline Headache/ post-ocular pain & 79 & 13.1 & Cerebral hemorrhage & 3 \\
\hline Ophthalmoplegia & 73 & 12.1 & Trigeminal nerve palsy & 3 & 0.5 \\
\hline Fatigue & 33 & 5.5 & Orthostatic hypotension & 3 \\
\hline Anorexia/ nausea & 27 & 4.5 & Amenorrhea/ galactorrhea & 2 \\
\hline Hyperprolactinemia & 27 & 4.5 & Cushing's syndrome & 0.5 \\
\hline Pituitary apoplexy & 10 & 1.7 & Acromegaly & 0.3 \\
\hline Altered mental state & 6 & 1.0 & CSWS & 2 \\
\hline Cognitive/ psychiatric deficit & 5 & 0.8 & Tumor growth on & 0.3 \\
\hline
\end{tabular}

N.B. SIADH: Syndrome of Inappropriate Antidiuretic Hormone; CSWS: Cerebral Salt Wasting Syndrome.

Table 2: Clinical presentation of 290 symptomatic pituitary metastases.

\begin{tabular}{|c|c|c|c|c|c|}
\hline Radiological Feature & $\mathbf{n}$ & $\%$ & Radiological Feature & $\mathbf{n}$ & $\%$ \\
\hline Sellar enhancing mass & 120 & 72.3 & Sphenoid sinus invasion & 21 & 12.7 \\
\hline Chiasm/ hypothalamus invasion & 62 & 37.3 & Loss of PL high signal intensity on T1W & 18 & 10.8 \\
\hline Stalk enhancing/ thickening & 52 & 31.3 & Other brain metastatic lesions & 17 & 10.2 \\
\hline Cavernous sinus invasion & 50 & 30.1 & Retrosellar enhancing mass & 9 & 5.4 \\
\hline Sellar abnormality/ enlargement & 47 & 28.3 & Low-intensity signal on T2W & 8 & 4.8 \\
\hline Dumbbell shaped mass & 46 & 27.7 & Negative & 1 & 0.6 \\
\hline Heterogeneous/ ring tumor enhancement & 35 & 21.1 & & & \\
\hline
\end{tabular}

N.B. PL: posterior lobe; T1W: T1-weighted sequence; T2W: T2 weighted sequence.

Table 3: MRI features of 166 symptomatic pituitary metastasis.

the affinity for such tumors to involve the posterior pituitary early in the disease course. Some have even suggested that early CDI to be the most important symptom differentiating metastasis from pituitary adenomas $[3,7,8]$. The clinician must be aware that untreated hypocortisolism, as in patient 2, could mask underlying CDI since cortisol reduces renal free water clearance [4]. In addition, ophthalmoplegia on presentation also suggests aggressive infiltration of the cavernous sinus. All three of our patients presented with relatively short durations of visual deterioration ranging from a few days to a month with two developing ophthalmoplegia during this period. In comparison only 5\% of patients with pituitary adenomas present with this symptom [9]. In rare instances, as in patient 1 , pituitary metastases can become the first manifestation of an occult malignancy occurring in fewer than $10 \%$ of cases [10]. An analysis of the MRI features of 160 reported cases indicates an absence of pathognomonic radiological signs (Table 3 ). Unless concurrent metastatic brain tumors are observed, which is a relatively uncommon occurrence (10.4\%) imaging interpretation can be challenging. Yet some characteristics may be suggestive including:
1. thickened pituitary stalk ( $31.3 \%) ; 2$. invasion of the cavernous sinus (30.1\%); 3. heterogeneous (or ring) contrast enhancement $(21.1 \%)$; and 4 . loss of the normal hyperintense signal of the posterior pituitary lobe on T1 weighted sequences (T1W) (10.8\%). Predominant posterior pituitary involvement is perhaps the most important distinguishing feature in differentiating metastasis from adenoma (Figure 1a and e-g) $[3,4,11]$. There is some controversy as to what constitutes a thickened pituitary stalk, but recent MRI studies have determined that the normal adult anteroposterior diameter at the level of its insertion into the pituitary to be $2.3 \pm 0.4 \mathrm{~mm}$ [12]. Cavernous sinus invasion also occurs more frequently in pituitary metastasis, but is only demonstrated in $6 \%$ to $10 \%$ of pituitary adenomas [13]. It has been suggested that early stage metastatic lesions are dumbbell shaped due to constriction by the diaphragm sellae (Figure 1h) and over a quarter of lesions (27.7\%) are of this morphology $[3,4]$. Overall survival of patients with pituitary metastasis is poor ranging from six to 22 months [3]. Surgical resection is often reserved for palliative purposes, to alleviate visual or intractable headache symptoms, and has not been proven to prolong survival [14]. 
Citation: Peter YMW, Ronald L, Yung C, Timothy SKC, Peter KHP, et al. (2015) Symptomatic Metastasis to the Pituitary Gland: A Report of Three Cases and Review of the Literature. J Neurol Disord 3: 236. doi: 10.4172/2329-6895.1000236

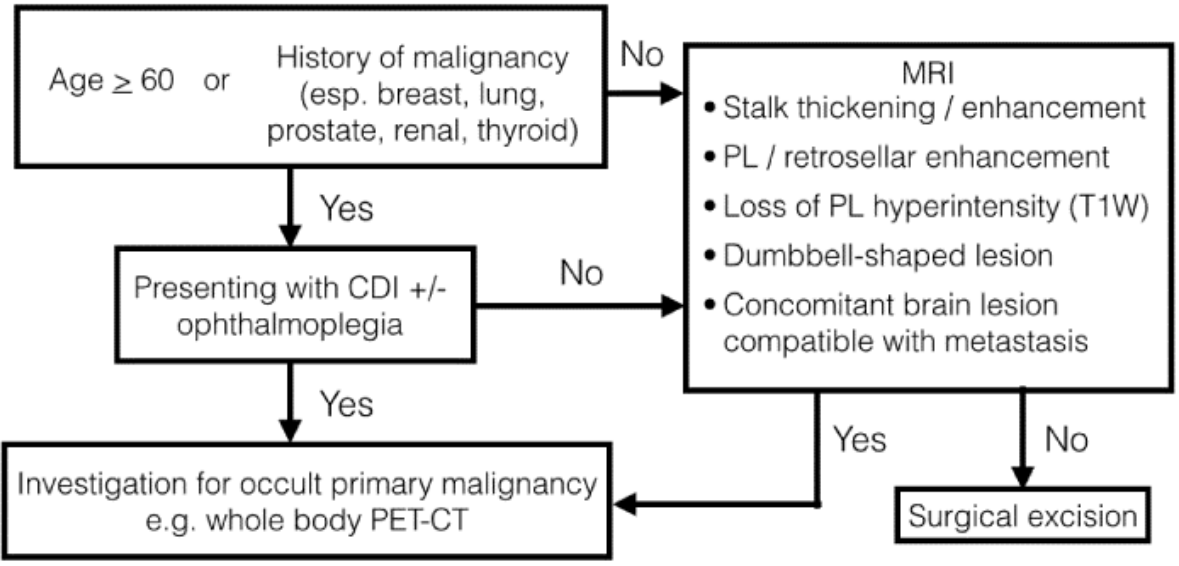

Figure 2: Diagnostic strategy for patients with suspected pituitary metastasis N.B. CDI: Cranial Diabetes Insipidis; MRI: Magnetic Resonance Imaging; PL: Posterior Pituitary Lobe; T1W: T1-Weighted Sequence; PET-CT: Positron-Emission Tomography Computed Tomography Scan.

It is imperative to distinguish pituitary metastasis from adenomas to avoid unnecessary surgery. An effective therapeutic alternative in selected symptomatic patients is radiosurgery. One case series adopting the Leksell Gamma Knife system (Elekta Inc, Atlanta, Ga) successfully relieved DI and neurological symptoms in $40 \%$ and $50 \%$ of patients respectively [10]. In light of our findings we propose that patients older than 60 years, or those with a history of cancer, presenting with DI or ophthalmoplegia along with supporting MRI evidence should be investigated for an occult malignancy (Figure 2).

\section{Conclusion}

The pituitary gland is a rare site for metastasis, but clinicians should be cognizant of this important diagnosis. Discussions on a comprehensive diagnostic strategy established by reviewing the existing literature are limited. Current understanding of pituitary metastasis is largely restricted to case reports and are indispensible given their rarity. However, the heterogeneity of reported clinicoradiological information poses challenges for in-depth analysis. For example, only 57\% of documented patients with symptomatic pituitary metastasis have their MRI described. In spite of this limitation we offer an updated literature review of available data and propose several red flags that should alert the clinician. Old age, a history of cancer, CDI or ophthalmoplegia upon presentation are important clinical features. When the MRI reveals predominantly posterior pituitary abnormal signal changes further investigations for an occult primary malignancy is recommended.

\section{References}

1. Saeger W, Lüdecke DK, Buchfelder M, Fahlbusch R, Quabbe HJ, et al. (2007) Pathohistological classification of pituitary tumors: 10 years of experience with the German Pituitary Tumor Registry. Eur J Endocrinol 156: 203-216.
2. Teears RJ, Silverman EM (1975) Clinicopathologic review of 88 cases of carcinoma metastatic to the putuitary gland. Cancer 36: 216-220.

3. Komninos J, Vlassopoulou V, Protopapa D, Korfias S, Kontogeorgos G, et al. (2004) Tumors metastatic to the pituitary gland: case report and literature review. J Clin Endocrinol Metab 89: 574-580.

4. Fassett DR, Couldwell WT (2004) Metastases to the pituitary gland. Neurosurg Focus 16: E8.

5. Chiang MF, Brock M, Patt S (1990) Pituitary metastases. Neurochirurgia (Stuttg) 33: 127-131.

6. Morita A, Meyer FB, Laws ER Jr (1998) Symptomatic pituitary metastases. J Neurosurg 89: 69-73.

7. Hermet M, Delévaux I, Trouillier S, André M, Chazal J, et al. (2009) Pituitary metastasis presenting as diabetes insipidus: a report of four cases and literature review. Rev Med Interne 30: 425-429.

8. Gormally JF, Izard MA, Robinson BG, Boyle FM (2014) Pituitary metastasis from breast cancer presenting as diabetes insipidus. BMJ Case Rep 2014

9. Chuang CC, Chen E, Huang YC, Tu PH, Chen YL, et al. (2011) Surgical outcome of oculomotor nerve palsy in pituitary adenoma. J Clin Neurosci 18 : 1463-1468.

10. Kano H, Niranjan A, Kondziolka D, Flickinger JC, Lunsford LD (2009) Stereotactic radiosurgery for pituitary metastases. Surg Neurol 72: 248-255.

11. Schubiger O, Haller D (1992) Metastases to the pituitary--hypothalamic axis. An MR study of 7 symptomatic patients. Neuroradiology 34: 131-134.

12. Satogami N, Miki Y, Koyama T, Kataoka M, Togashi K (2010) Normal pituitary stalk: high-resolution MR imaging at 3T. AJNR Am J Neuroradiol 31: 355-359.

13. Cottier JP, Destrieux C, Brunereau L, Bertrand P, Moreau L, et al. (2000) Cavernous sinus invasion by pituitary adenoma: MR imaging. Radiology 215: 463-469.

14. Zoli M, Mazzatenta D, Faustini-Fustini M, Pasquini E, Frank G (2013) Pituitary metastases: role of surgery. World Neurosurg 79: 327-330. 